\title{
Avaliação das propriedades mecânicas de quatro cimentos de ionômero de vidro convencionais utilizados na cimentação de bandas ortodônticas
}

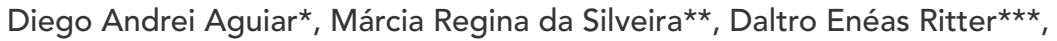

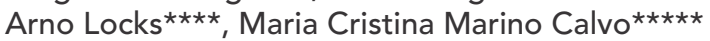

\begin{abstract}
Resumo
Objetivo: objetivo deste estudo foi comparar a resistência flexural, resistência à compressão e resistência à tração diametral de quatro diferentes cimentos de ionômero de vidro convencionais utilizados para cimentação de bandas ortodônticas (Vidrion C/SS White; Meron/Voco; Ketac Cem/3M ESPE; Vivaglasss/Ivoclar-Vivadent). Metodologia: foram confeccionados 12 corpos-de-prova para cada cimento, em cada teste, seguindo a norma 66 da ADA para resistência à tração diametral e à compressão, e norma 4049 da ISO para resistência flexural. Os testes foram feitos após 24 horas de armazenagem em água destilada a $37^{\circ} \mathrm{C}$, na máquina de testes Universal Instron 4444 sob velocidade de $0,75 \mathrm{~mm} /$ minuto para resistência flexural e $1 \mathrm{~mm} / \mathrm{min}$ para resistência à tração diametral e à compressão. Os resultados obtidos para o teste de resistência flexural foram: 25,85 $\pm 5,43 \mathrm{MPa}$ (Meron); 21,85 $\pm 6,96 \mathrm{MPa}$ (Vidrion C); $20,85 \pm 4,17 \mathrm{MPa}$ (Vivaglass); 20,50 $\pm 4,89 \mathrm{MPa}$ (Ketac Cem). Para o teste de compressão os resultados foram (na mesma ordem): 77,72 $\pm 20,21 \mathrm{MPa} ; 56,49 \pm 8,54 \mathrm{MPa} ; 47,84 \pm 9,25 \mathrm{MPa}$; $81,93 \pm 13,37 \mathrm{MPa}$. Resultados: os resultados para o teste de resistência à tração diametral foram (na mesma ordem): 9,59 $\pm 2,09 \mathrm{MPa} ; 5,25 \pm 1,29 \mathrm{MPa} ; 7,69 \pm 2,09 \mathrm{MPa} ; 4,08 \pm 1,32 \mathrm{MPa}$. Resultados: os quatro cimentos mostraram-se estatisticamente equivalentes quanto ao teste de resistência flexural. Os cimentos Meron e Ketac Cem foram estatisticamente mais resistentes à compressão que o Vidrion $\mathrm{C}$ e o Vivaglass. Em relação ao teste de resistência à tração diametral, o cimento Meron mostrou-se estatisticamente superior aos demais.
\end{abstract}

Palavras-chave: lonômero de vidro. Bandas. Cimentação.

\footnotetext{
* Cirurgião-dentista, aluno do curso de especialização em Ortodontia da Universidade Federal de Santa Catarina.

** Mestre em Materiais Dentários UFSC. Especialista em Prótese e oclusão pela EAP ABO-SC.

*** Especialista e Mestre em Ortodontia UERJ-RJ. Doutor em Ortodontia UNESP Araraquara-SP. Professor Substituto de Ortodontia da UFSC.

**** Mestre e Doutor em Ortodontia pela UFRJ-RJ e UNESP - Araraquara. Pós-Doutorado em Ortodontia no Royal Dental College of Aarhus-Dinamarca. Coordenador do curso de Especialização em Ortodontia da UFSC.

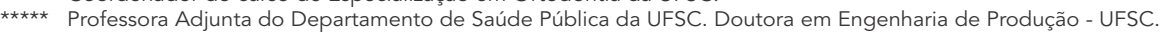




\section{INTRODUÇÃO}

Apesar do desenvolvimento e aprimoramento cada vez maior dos sistemas adesivos poliméricos utilizados nas colagens de peças ortodônticas, as bandas ainda constituem artifícios muito usados dentro da Ortodontia.

A retenção das bandas é influenciada mecanicamente pela sua adaptação ao dente e também pelo cimento utilizado. Para isto, existem os mais variados tipos de cimentos, todos com suas vantagens e limitações. Durante muitos anos o cimento mais utilizado foi o cimento de fosfato de zinco, que acabou em desuso por ser muito solúvel em boca e por contar inteiramente com a retenção mecânica, limitando muito seu sucesso clínico 9,10,11.

O cimento de policarboxilato de zinco, que surgiu no começo dos anos 70 , reage quimicamente com o esmalte, através da quelação de íons cálcio e possui, ainda, a capacidade de unir-se ionicamente com o aço inoxidável ${ }^{21}$. Porém, possui sérias limitações, como a alta viscosidade, curto tempo de trabalho e alta solubilidade em boca, mostrando não ser o mais adequado para a cimentação de bandas ortodônticas ${ }^{6}$.

Atualmente, o cimento mais utilizado para este fim tem sido o cimento de ionômero de vidro, que tem como principais vantagens a liberação de flúor, a inibição microbiana e a adesão química tanto com o dente quanto com o metal ${ }^{19}$. Os cimentos de ionômero de vidro (CIVs) foram inicialmente desenvolvidos por Wilson e Kent, no final dos anos $60^{18}$ e são formados a partir de uma reação ácidobase. Suas propriedades favoráveis de adesão e liberação de fluoretos os levaram a ser amplamente utilizados na Odontologia ${ }^{4,25}$ e mais recentemente como agentes cimentantes ${ }^{14}$. Essa capacidade do CIV em liberar fluoretos faz com que ocorra remineralização do tecido dentário que cerca o material ${ }^{14}$. Porém, os materiais restauradores atuais com alta liberação de fluoretos, geralmente, têm propriedades mecânicas mais baixas ${ }^{23}$, como baixa resistência ao desgaste, fragilidade e baixa dureza ${ }^{7}$. Além disso, os CIVs, absorvem ou perdem água do ambiente nas fases iniciais da cura, o que pode enfraquecer suas propriedades mecânicas ${ }^{25}$. Já as resinas compostas e alguns compômeros, que são mecanicamente mais fortes, liberam apenas uma pequena quantidade de fluoretos ${ }^{23}$. Atualmente, ionômeros de vidro modificados por resina parecem oferecer o melhor equilíbrio entre liberação de flúor, recaptação de fluoretos do meio bucal e maior durabilidade clínica para pacientes com alto risco de cárie ${ }^{23}$. Além disso, são fotopolimerizáveis (maior controle do tempo de trabalho) e alcançam resistência máxima em um período de tempo menor'.

O objetivo desta pesquisa foi avaliar a resistência à compressão, a resistência à tração diametral e a resistência flexural de 4 cimentos de ionômero de vidro convencionais, utilizados para a cimentação de bandas ortodônticas.

\section{MATERIAL E MÉTODOS Material}

Os cimentos que foram submetidos aos testes mecânicos estão especificados na tabela 1 , com o número de série e fabricante, respectivamente.

\section{Preparação das amostras}

Para o teste de resistência flexural, doze corpos-de-prova por cimento foram confeccionados a partir de uma matriz metálica bipartida medindo $25 \mathrm{~mm}( \pm 2 \mathrm{~mm})$ de comprimento por $2 \mathrm{~mm}$ $( \pm 0,1 \mathrm{~mm})$ de largura e altura (Fig. $1 \mathrm{~A}, \mathrm{~B})$, seguindo as normas da ISO (International Organization for Standardization), padrão $4049^{5}$.

Tabela 1 - Relação dos materiais e fabricantes utilizados na pesquisa.

\begin{tabular}{ccc}
\hline Material & Número de série & Fabricante \\
\hline Meron & Lot. 460314 & Voco \\
Vivaglass Cem PL & Lot. 97508 & Ivoclar Vivadent \\
Vidrion C & Lot. 03177 & SS White \\
Ketac Cem Easy mix & Lot. 162094 & 3M-ESPE \\
\hline
\end{tabular}




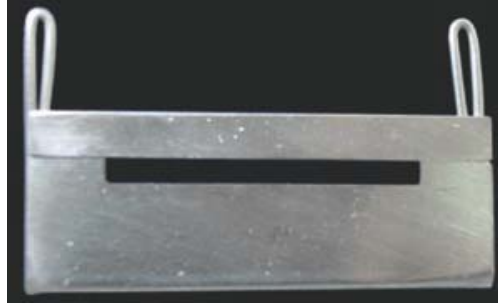

FIGURA 1A - Matriz metálica bipartida utilizada para confecção dos corpos-de-prova para o teste de resistência flexural.

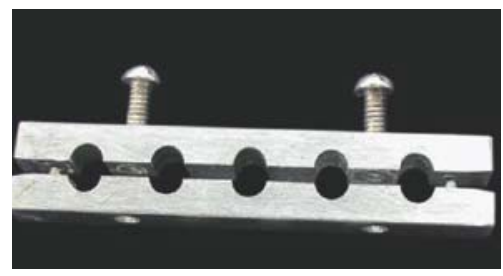

FIGURA 2A - Matriz metálica bipartida utilizada para confecção dos corpos-de-prova para o teste de resistência à compressão e resistência à tração diametral.

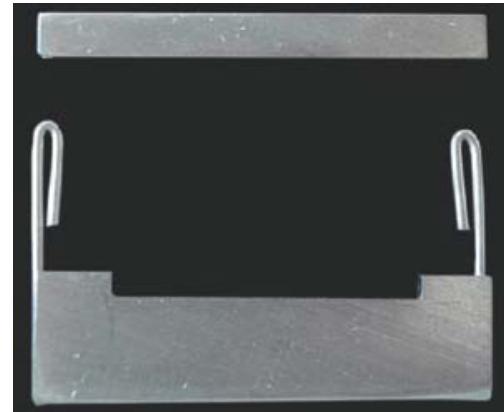

FIGURA 1B - Matriz metálica bipartida entreaberta utilizada para confecção dos corpos-de-prova para o teste de resistência flexural.

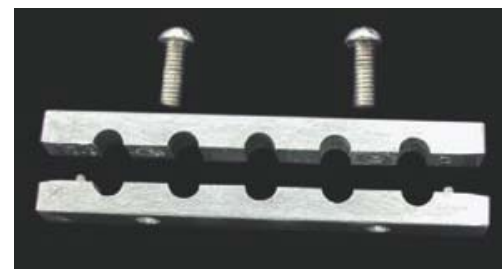

FIGURA 2B - Mesma matriz metálica bipartida entreaberta.

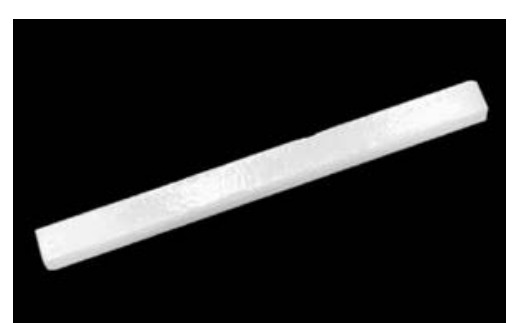

FIGURA 1C - Corpo-de-prova para do teste de resistência flexural.

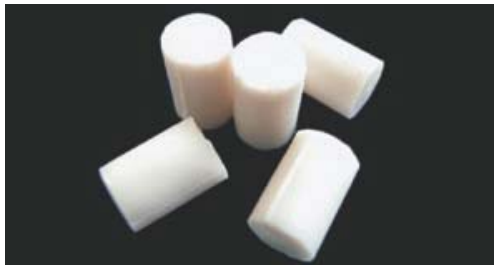

FIGURA 2C - Corpos-de-prova dos testes de resistência à compressão e tração diametral.
A matriz foi posicionada sobre uma lâmina de vidro interposta por uma película de poliéster. O material foi manipulado de acordo com as recomendações do fabricante e inserido na matriz. Outra lâmina de vidro foi colocada no topo do molde, exercendo-se uma leve pressão para o escoamento de excesso de material. Esperou-se o tempo de presa recomendado pelo fabricante e, em seguida, as amostras, unidas à matriz, foram mergulhadas em água destilada a $37^{\circ} \mathrm{C}$ por 15 minutos. Após os 15 minutos, as amostras foram aparadas e polidas com lixa de granulação 600 (Fig. 1C) e armazenadas em água destilada a $37^{\circ} \mathrm{C}$, por 24 horas.

Doze corpos-de-prova cilíndricos por cimento foram confeccionados para o teste de resistência à compressão e doze corpos-de-prova cilíndricos por cimento foram confeccionados para o teste de resistência à tração diametral, todos a partir de uma matriz metálica bipartida, com dimensões de $6 \mathrm{~mm}$ de altura por $4 \mathrm{~mm}$ de diâmetro (Fig. 2A, B), de acordo com especificação ADA n ${ }^{\circ} 66$ de 1994.

A matriz foi posicionada sobre uma placa de vidro interposta por uma tira de poliéster. Os cimentos foram manipulados com uma espátula plástica, sobre uma placa de vidro, na proporção pó-líquido de acordo com as recomendações dos fabricantes. O cimento foi, então, inserido na matriz e uma nova tira de poliéster foi posicionada. Sobre este conjunto uma lamínula de vidro foi comprimida a fim de se extravasar os excessos. Foi aguardado o tempo de presa final, recomendado por cada fabricante, para se separar as amostras. As amostras foram, então, aparadas e polidas com lixa de granulação 600 (Fig. 2C) e armazenadas em água destilada a $37^{\circ} \mathrm{C}$, por 24 horas, até o momento do teste. 


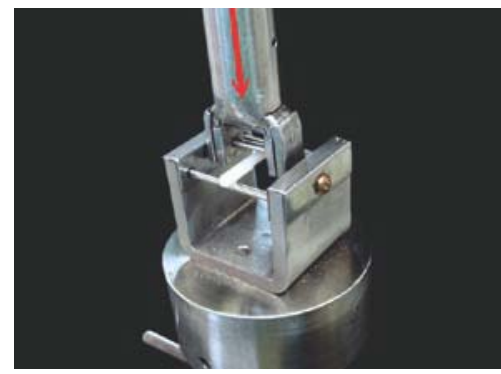

FIGURA 3 - Teste de resistência flexural. Corpo-deprova com seu longo eixo no sentido horizontal.

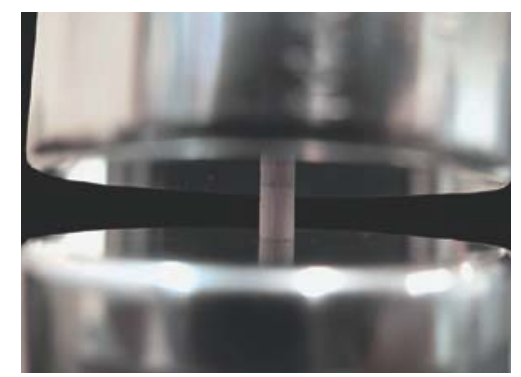

FIGURA 4 - Teste de resistência à compressão. Corpo-de-prova com seu longo eixo no sentido vertical.

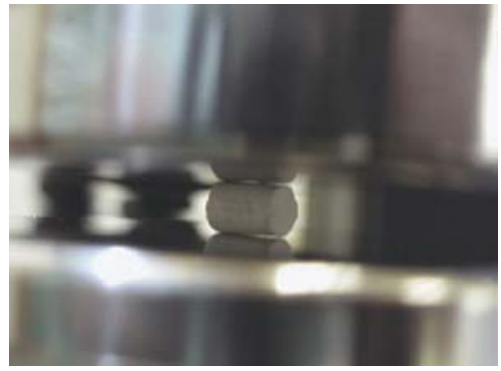

FIGURA 5 - Teste de resistência à tração diametral. Corpo-de-prova com seu longo eixo no sentido horizontal.

\section{Teste de resistência flexural}

A resistência flexural do cimento de ionômero de vidro convencional foi determinada através do teste de 3 pontos em uma Máquina de Testes Universal Instron, modelo 4444 (Instron Corp, Canton, Mass, USA). As peças para a realização do teste estavam de acordo com as normas da ISO 4049. Uma das peças era composta por uma base contendo dois cilindros de $2 \mathrm{~mm}$ de diâmetro, paralelos e distantes $20 \mathrm{~mm}$, sobre os quais posicionava-se o corpo-de-prova. Para a fratura da amostra, uma outra peça contendo um terceiro cilindro, também medindo $2 \mathrm{~mm}$ de espessura, descia a uma velocidade de $0,75 \mathrm{~mm} / \mathrm{min}$ sobre o centro do espécime, até sua fratura (Fig. 3). Os valores de fratura eram fornecidos por um painel anexo à máquina de ensaios. A resistência flexural foi calculada, em MPa, através da seguinte equação:

Resistência flexural $(\mathrm{MPa})=3 \mathrm{Fl} / 2 \mathrm{bh}^{2}$

Onde $\mathrm{F}$ foi a carga máxima exercida na amostra $(\mathrm{N}), 1$ a distância entre os suportes (mm), b a largura da amostra $(\mathrm{mm})$ e h a altura da amostra $(\mathrm{mm})$.

\section{Teste de resistência à compressão e à tração diametral}

Os corpos-de-prova para os testes de resistência à tração diametral e à compressão foram testados na mesma máquina já citada. As peças para estes testes constituíam-se de 2 pratos, um fixo que servia como base e outro móvel que descia ao encontro do fixo a uma velocidade de $1 \mathrm{~mm} / \mathrm{seg}$. A diferença entre os dois testes é que no teste de compressão o corpo-de-prova foi posicionado com seu longo eixo no sentido vertical (Fig. 4) e no de tração diametral o longo eixo foi posicionado no sentido horizontal (Fig. 5).

A tensão de compressão foi calculada, em Mpa, pela fórmula que segue:

Tensão de compresão $(\mathrm{MPa})=4 \mathrm{~F} / \pi \mathrm{d}^{2}$

Onde $\mathrm{F}$ foi a carga de ruptura $(\mathrm{N})$ e d o diâmetro da amostra.

Para se calcular a tração diametral (MPa) aplicou-se a seguinte fórmula:

Tensão de tração diametral $(\mathrm{MPa})=2 \mathrm{~F} / \pi \mathrm{d} l$

Onde F foi a carga de ruptura $(\mathrm{N})$, d o diâmetro da amostra (mm) e l o comprimento da amostra $(\mathrm{mm})$.

\section{RESULTADOS}

A análise estatística descritiva (média e desviopadrão) para o teste de resistência flexural encontra-se na tabela 2.

Tabela 2 - Valores de média e desvio-padrão para o teste de resistência flexural. Não houve diferença estatisticamente significante para os quatro cimentos $(p>0,05)$.

\begin{tabular}{cccc}
\hline material & $\mathbf{n}$ & média & d.p. \\
\hline Meron & 12 & 25,85 & 5,43 \\
Vidrion & 12 & 21,85 & 6,96 \\
Vivaglass & 12 & 20,85 & 4,17 \\
Ketac Cem & 12 & 20,50 & 4,89 \\
\hline
\end{tabular}


Aplicou-se o teste de análise de variância de 1 critério (ANOVA 1) e a estatística mostrou não haver diferença significante $(\mathrm{p}>0,05)$ entre os quatro materiais para o teste de resistência flexural (Gráf. 1).

Os resultados para o teste de resistência à compressão estão listados na tabela 3. Ao serem detectadas diferenças no teste ANOVA ( $p<0,0001)$, foi aplicado o teste de Tukey para comparações individuais. Os cimentos Meron e Ketac Cem apresentaram valores de resistência à compressão estatisticamente superiores ao Vidrion e o Vivaglass (Gráf. 2).

A tabela 4 mostra os resultados para o teste de resistência à tração diametral. Houve diferença estatisticamente significante entre os materiais $(\mathrm{p}<$ 0,0001). O cimento Meron foi superior aos demais.

Tabela 3 - Valores de média e desvio-padrão para o teste de resistência à compressão. 0 cimento Meron e o Ketac Cem não apresentaram diferença estatisticamente significante entre si $(p>0,05)$, mas apresentaram diferença estatisticamente significante em relação aos demais $(p<0,0001)$. Vidrion e Vivaglass não apresentaram diferença entre si ( $p>0,05)$.

\begin{tabular}{cccc}
\hline material & $\mathbf{n}$ & média & d.p. \\
\hline Ketac Cem & 12 & 81,93 & 13,37 \\
Meron & 12 & 77,72 & 20,21 \\
Vidrion & 12 & 56,49 & 8,54 \\
Vivaglass & 12 & 47,84 & 9,25 \\
\hline
\end{tabular}

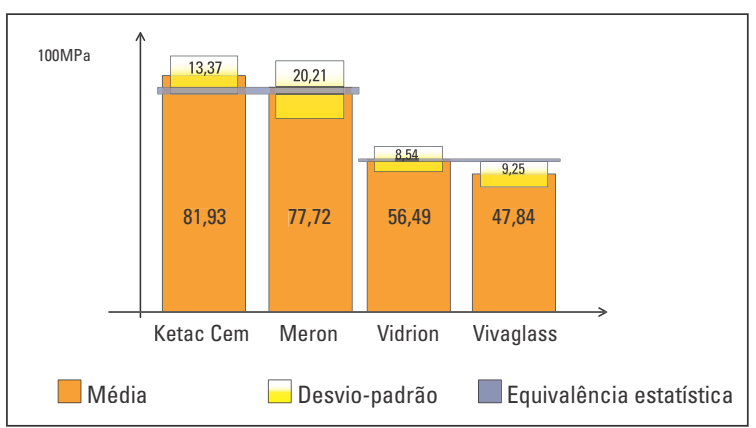

GRÁFICO 2 - Representação gráfica dos valores médios de resistência à compressão e desvio-padrão encontrados para os 4 cimentos testados. Os cimentos Ketac Cem e Meron foram estatisticamente equivalentes entre si e superiores $(p<0,0001)$ aos cimentos Vidrion $C$ e Vivaglass, que foram equivalentes entre si.

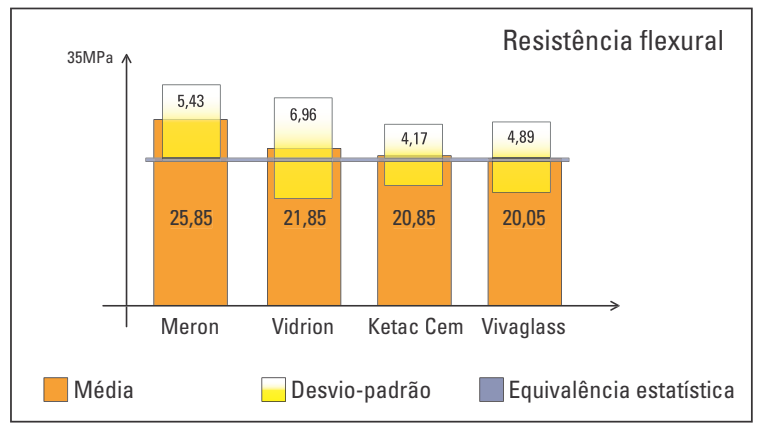

GRÁFICO 1 - Representação gráfica dos valores médios e desvios-padrão encontrados para os 4 cimentos testados. Todos os cimentos foram estatisticamente equivalentes $(p>0,05)$.

O cimento Vivaglass, que foi menos resistente que o Meron, apresentou-se superior aos cimentos Vidrion e Ketac Cem. Os dois últimos não apresentaram diferença estatística entre si (Gráf. 3).

Tabela 4 - Valores de média e desvio-padrão para o teste de resistência à tração diametral. 0 cimento Meron apresentou diferença estatisticamente significante em relação aos demais $(p<0,0001)$. 0 cimento Vivaglass apresentou diferença estatisticamente significante em relação ao Vidrion e Ketac Cem $(p<0,0001)$ que não apresentaram diferenças entre si $(p>0,05)$.

\begin{tabular}{cccc}
\hline material & $\mathbf{n}$ & média & d.p. \\
\hline Meron & 12 & 9,59 & 2,09 \\
Vivaglass & 12 & 7,69 & 2,09 \\
Vidrion & 12 & 5,25 & 1,29 \\
Ketac Cem & 12 & 4,08 & 1,32 \\
\hline
\end{tabular}

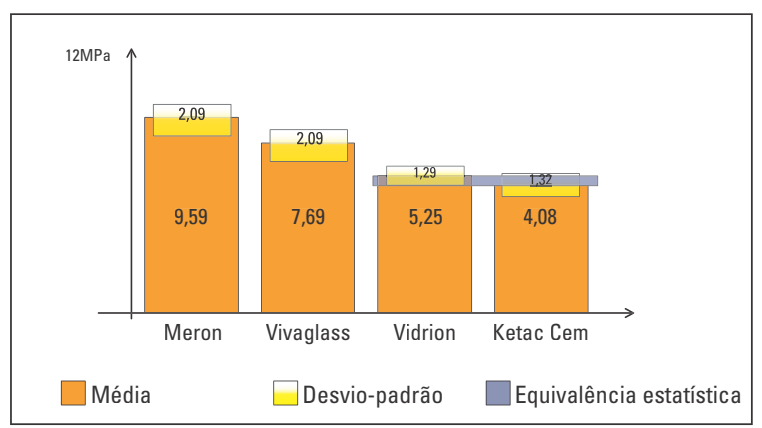

GRÁFICO 3 - Representação gráfica dos valores médios de resistência à tração diametral e desvio-padrão encontrados para os 4 cimentos testados. 0 cimento Meron foi estatisticamente superior $(p<0,0001)$ aos demais. Vivaglass foi superior ao Vidrion e Ketac Cem, que entre si foram equivalentes. 


\section{DISCUSSÃO}

A desmineralização do esmalte tem registros de prevalência de até $96 \%$ em pacientes que fazem uso de terapia com aparelhos fixos ${ }^{12}$. A alta prevalência, aliada às propriedades biológicas e anticariogênicas dos ionômeros de vidro, foi decisiva para que esse material se tornasse o agente mais utilizado na cimentação de bandas ortodônticas.

Os ionômeros de vidro, entretanto, não possuem propriedades mecânicas muito elevadas, o que leva a certas falhas clínicas quando, após a cimentação, forças incidem sobre a banda. $\mathrm{O}$ agente utilizado como cimento deve ser capaz de resistir a essas forças, mantendo a banda estável em boca, contribuindo para o sucesso do tratamento. Os cimentos de ionômero de vidro utilizados para cimentação têm a desvantagem de serem muito sensíveis à umidade durante a presa, com um ganho de resistência muito tardio, devido ao policarboxilato de alumínio, que leva 24 horas para ser formado e é o responsável pelo aumento das propriedades mecânicas dos cimentos ${ }^{15,17}$. Como principais vantagens, os CIVs apresentam a liberação de fluoretos e adesão à estrutura dentária ${ }^{9,10}$.

As composições dos cimentos de ionômero de vidro testados nesta pesquisa são muito semelhantes. O pó é composto principalmente por ácido poliacrílico (que confere resistência ao cimento), partículas inorgânicas e pigmentos. O líquido é composto, basicamente, de ácido tartárico, que funciona como quelante de íons e acelerador da reação química e água.

Os resultados encontrados para o teste de resistência flexural foram semelhantes para os quatro cimentos (Tab. 2, Gráf. 1). Esse teste tem como principal vantagem o estado de tensão pura que pode ser estabelecido sobre um lado do espécime. Ele mensura simultaneamente as tensões de tração, compressão e cisalhamento ${ }^{2}$, simulando as condições desenvolvidas na cavidade bucal. Embora não tenha havido superioridade estatística de nenhum cimento de ionômero de vidro, os valores encontrados para este teste foram muito baixos, caracterizando uma grande desvantagem em relação aos CIV modificados por resina, que apresentam, normalmente, valores de resistência flexural duas vezes maiores que os cimentos convencionais $^{20,22}$.

O teste de resistência à compressão permite uma boa representação da integridade mecânica dos materiais, sendo usado para testar materiais friáveis, como os cimentos de ionômero de vi$\mathrm{dro}^{13}$. Esta propriedade é extremamente importante, particularmente no processo de mastigação. Neste teste, duas forças axiais são aplicadas ao material em direções opostas, aproximando sua estrutura molecular ${ }^{16}$. Os cimentos Meron e Ketac Cem apresentaram-se estatisticamente superiores aos demais (Tab. 3, Gráf. 2). Somente estes dois cimentos atingiram os valores mínimos recomendados pela ADA, que estabelece que os cimentos para cimentação devem apresentar um valor mínimo de resistência à compressão de $65 \mathrm{Mpa}^{1}$. A literatura reporta que os valores para esta propriedade não diferem entre os ionômeros de vidro convencionais e os modificados por resina ${ }^{25}$.

A maioria das falhas clínicas dos materiais ocorre devido à sua baixa resistência à tração ${ }^{8}$. O teste de resistência à tração diametral é uma adaptação do teste de resistência à tração, por ser impossível a realização deste em materiais friáveis como os cimentos de ionômeros de vidro ${ }^{3}$. Nesse teste, uma força compressiva é aplicada a um espécime cilíndrico sobre o seu diâmetro. Os cimentos Meron e Vivaglass obtiveram resultados superiores estatisticamente em relação aos demais cimentos (Tab. 4, Gráf. 3). Estes resultados indicam que estes cimentos são os que mais resistem às cargas de fratura, indicando um maior valor da força coesiva do material.

Para todos os cimentos, os valores de resistência à compressão foram muito superiores aos de resistência à tração diametral. Os valores de compressão foram mais do que nove vezes superiores aos valores de resistência à tração diametral. Resultados semelhantes foram encontrados na pesquisa 
de Yap, Pek e Cheang ${ }^{24}$, que reportaram ainda que após uma semana de armazenagem os valores de resistência à compressão foram superiores aos armazenados por 24 horas.

Por isso, é extremamente importante que o ortodontista conheça as propriedades do material que utiliza em seu consultório, sabendo não apenas de suas vantagens, mas também de suas limitações. É necessário seguir à risca as recomendações do fabricante, para que se possa usufruir ao máximo das qualidades do material escolhido. O estudo comparativo, in vitro, dos cimentos de ionômero de vidro convencionais não reproduz com exatidão todas as variáveis existentes em boca. Assim, o simples fato de um cimento ser mecanicamente mais resistente que o outro não lhe confere garantia de sucesso clínico.

\section{CONCLUSÃO}

De acordo com os testes realizados na amostragem, os resultados evidenciaram estatisticamente que:

1) Os quatro cimentos foram equivalentes no teste de resistência flexural;

2) Os cimentos Meron e Ketac Cem mostraram-se mais resistentes à compressão que o Vidrion C e o Vivaglass;

3) No teste de resistência à tração diametral, o Meron foi superior aos demais cimentos.

\title{
Evaluation of mechanical properties of four conventional glass ionomer cements used in orthodontic bands cementation
}

\begin{abstract}
Aim: The aim of this in vitro study was to compare the flexural strength, the compressive strength and the diametral tensile strength of four conventional glass-ionomer cements (Vidrion C/SS White; Meron/Voco; Ketac Cem/3M ESPE; Vivaglass/lvoclar-Vivadent) used in Orthodontics for band cementation. Methods: Twelve specimens of each cement were prepared for each test. Specimens were fabricated according to ISO 4049 (flexural strength) and to the ADA specification \#66 (compressive strength and diametral tensile strength). Specimens were stored in distilled water at $37^{\circ} \mathrm{C}$ for 24 hours, and tested using an Instron Universal Testing Machine 4444 with crosshead speeds of $0.75 \mathrm{~mm} /$ minute (flexural strength) and $1 \mathrm{~mm} /$ minute (compressive strength and diametral strength). Results: The results (mean $\pm \mathrm{SD}$, in MPa) for flexural strength were: $25.85 \pm 5.43$ (Meron); $21.85 \pm 6.96$ (Vidrion C); $20.85 \pm$ 4.17 (Vivaglass); $20.50 \pm 4.89$ (Ketac Cem). Compressive strength (in the same order): $77.72 \pm 20.21 ; 56.49 \pm 8.54$; $47.84 \pm 9.25 ; 81.93 \pm 13.37$. The results for diametral tensile strength were (in the same order): $9.59 \pm 2.09 ; 5.25$ $\pm 1.29 ; 7.69 \pm 2.09 ; 4.08 \pm 1.32$. Conclusions: No statistically significant difference was detected in the flexural strength among the different groups. Meron and Ketac Cem yielded greater mean compressive strength values than Vidrion $\mathrm{C}$ and Vivaglass. Meron resulted in significantly higher diametral tensile strength mean value than the other cements.
\end{abstract}

Key words: Glass ionomer. Band. Cementation. 


\section{REFERÊNCIAS}

1. AMERICAN NATIONAL STANDARD. Chicago: American Dental Association, 1994. Specification, 66.

2. BAN, S.; ANUSAVICE, K. J. Influence of test method on failure stress of brittle dental materials. J. Dent. Res., Alexandria, v. 69 , no. 12 , p. $1791-1799$, Dec. 1990.

3. BRITISH STANDARD SPECIFICATION FOR DENTAL GLASS IONOMER CEMENTS. Dental Standards Committee, BS 6039. London: British Standard Institution, 1981. p. 4.

4. GU, Y. W.; YAP, A. U.; CHEANG, P.; KUMAR, R. Spheroidization of glass powders for glass ionomer cements. Biomaterials, Oxford, v. 25, no. 18, p. 4029-4035, Aug. 2004.

5. INTERNATIONAL ORGANIZATION FOR STANDARDIZATION. ISO Standard 4049: Dentistry Polymer-based filling, restorative and luting materials. Geneva, 2000.

6. JOHSON, N. Current products and practice orthodontic banding cements. J. Orthod., London, v. 27, no. 3, p. 283-284, sept. 2000.

7. KLEVERLAAN, C. J.; VAN DUINEN, R. N. B.; FEILZER, A. J. Mechanical properties of glass ionomer cements affected by curing methods. Dent. Mater., Kidlington, v. 20, no. 1, p. 45-50, Jan. 2004.

8. MCKINNEY, J. E.; ANTONUCCI, J. M.; RUPP, N. W. Wear and microhardness of glass-ionomer cements. J. Dental Res., Alexandria, v. 66, p. 1134-1139, 1987.

9. MILLETT, D. T. et al. Resin-modified glass ionomer, modified composite or conventional glass ionomer for band cementation? An in vitro evaluation. Eur. J. Orthod., Oxford, v. 25, p. 609-614, 2003.

10. MILLETT, D. T. et al. In vitro comparison of orthodontic band cements. Am. J. Orthod. Dentofacial Orthop., St. Louis, v. 123, no. 1, p. 15-20, 2003.

11. MILLETT, D. T. et al. A clinical retrospective evaluation of 2 orthodontic band cements. Angle Orthod., Appleton, v. 71, no. 6, p. 470-476, 2001.

12. MITCHELL, L. Decalcification during orthodontic treatment with fixed appliances: an overview. Br. J. Orthod., Oxford, v. 19, no. 3, p. 199-205, 1992.

13. NAASAN, M. A.; WATSON, T. F. Conventional glass ionomers as posterior restorations: a status report for the American Journal of Dentistry. Am. J. Dent., San Antonio, v. 11, p. 36-45, 1998.
14. PALMER, G. et al. Chlorhexidine release from an experimental glass ionomer cement. Biomaterials, Oxford, v. 25, no. 23, p. 5423-5431, Oct. 2004.

15. PERSON, G. J.; ATKINSON, A. S. Long-term flexural strength of glass ionomer cements. Biomaterials, Oxford, v. 12, p. 658-660, 1991.

16. WANG, L. et al. Mechanical properties of dental restorative materials: relative contribution of laboratory tests. J. Appl. Oral Sci., Bauru, v. 11, p. 162-167, 2003.

17. WILLIAMS, J. A.; BILLINGTON, R. W. Changes in compressive strength of glass ionomer restorative materials with respect to time periods of $24 \mathrm{~h}$ to 4 months. J. Rehabil., Alexandria, v. 18, p. 163-168, 1991.

18. WILSON, A. D.; KENT, B. E. A new translucent cement for dentistry. Br. Dental J., London, v. 132, no. 4, p. 133-5, Feb. 1972.

19. WILSON, A. D.; PADDON, J. M.; CRISP, S. The hydration of dental cements. J. Dental Res., Alexandria, v. 58, p. 1065-1071, 1979

20. WILSON, A. D. Resin-modified glass-ionomer cements. Int. J. Prosthodont., Lombard, v. 3, p. 425-429, 1990.

21. WOOD, D. P.; PALECZNY, G. J.; JOHNSON, L. N. The effect of sandblasting on the retention of orthodontic bands. Angle Orthod., Appleton, v. 66, no. 3, p. 207-214, 1996.

22. XIE, D. et al. Mechanical properties and microstructures of glass-ionomer cements. Dent. Mater., Kidlington, v. 16, p. $129-138,2000$.

23. $X U, X$; BURGESS, J. O. Compressive strength, fluoride release and recharge of fluoride-releasing materials. Biomaterials, Oxford, v. 24, p. 2451-2461, 2003.

24. YAP, A.U. J.; PEK, Y. S.; CHEANG, P. Physico-mechanical properties of a fast-set highly viscous GIC restorative. J. Oral Rehabil., Oxford, v. 30, p. 1-8, 2003.

25. YLI-URPO, H. et al. Compressive strength and surface characterization of glass ionomer cements modified by particles of bioactive glass. Dent. Mater., Kidlington, v. 21, no. 3, p. 201-209, Mar. 2005.
Endereço para correspondência
Diego Andrei Aguiar
Rua Arno Hoeschel 55 ap 501 - Centro
CEP: $88.015-620$ - Florianópolis/SC
E-mail: drdiego@odontovip.com.br 